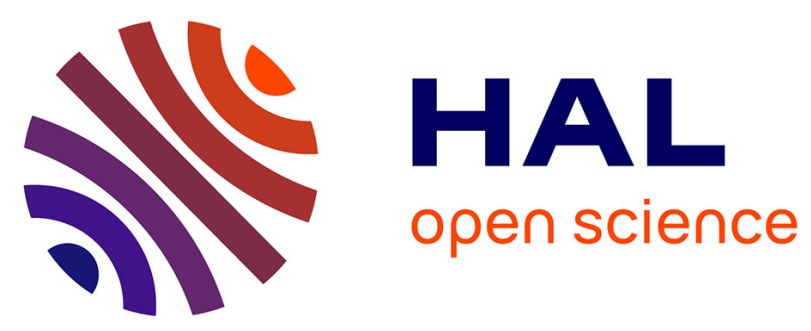

\title{
Quelle prise en charge optimale pour un sarcome du cordon spermatique en 2018 ?
}

S. Carrere, R. Tetreau, C. Honore, D. Tzanis, J.-B. Delhorme, M. Fau, G.

Decanter, C. Llacer, N. Firmin, E. Stoeckle, et al.

\section{- To cite this version:}

S. Carrere, R. Tetreau, C. Honore, D. Tzanis, J.-B. Delhorme, et al.. Quelle prise en charge optimale pour un sarcome du cordon spermatique en 2018 ?. Progrès en Urologie, 2019, 29 (1), pp.12-17. 10.1016/j.purol.2018.09.010 . hal-02462346

\section{HAL Id: hal-02462346 \\ https://hal.umontpellier.fr/hal-02462346}

Submitted on 21 Oct 2021

HAL is a multi-disciplinary open access archive for the deposit and dissemination of scientific research documents, whether they are published or not. The documents may come from teaching and research institutions in France or abroad, or from public or private research centers.
L'archive ouverte pluridisciplinaire HAL, est destinée au dépôt et à la diffusion de documents scientifiques de niveau recherche, publiés ou non, émanant des établissements d'enseignement et de recherche français ou étrangers, des laboratoires publics ou privés.

\section{(c) (1) $\$$}

Distributed under a Creative Commons Attribution - NonCommerciall 4.0 International 
Version of Record: https://www.sciencedirect.com/science/article/pii/S1 166708718305591

Manuscript_a4c6d2b418cd333baa09e754555f8da3

\section{Titre :}

Quelle prise en charge optimale pour un sarcome du cordon spermatique en 2018 ?

What is the best management for a spermatic cord sarcoma in 2018 ?

\section{Auteurs:}

Sébastien Carrère ${ }^{1}$, Raphael Tetreau ${ }^{2}$, Charles Honore ${ }^{3}$, Dimitri Tzanis ${ }^{3}$, Jean-Baptiste Delhorme $^{5}$, Magali Fau ${ }^{6}$, Gauthier Decanter ${ }^{7}$, Carmen Llacer ${ }^{8}$, Nelly Firmin ${ }^{9}$, Eberhard Stoeckle $^{10}$, Pierre Meeus ${ }^{11}$, Gwenaël Ferron ${ }^{12}$, François Quénet ${ }^{1}$, Bernard Meunier ${ }^{13}$, Sylvie Bonvalot ${ }^{4}$, GSF-GETO

\section{Affiliations:}

${ }^{1}$ Institut régional du Cancer de Montpellier (ICM), Service de chirurgie, 208 avenue des apothicaires, 34298 Montpellier, France

${ }^{2}$ Institut régional du Cancer de Montpellier (ICM), Service de radiologie, 208 avenue des apothicaires, 34298 Montpellier, France

${ }^{3}$ Institut Gustave Roussy (IGR), Service de chirurgie, 114 Rue Edouard Vaillant, 94800 Villejuif, France ${ }^{4}$ Institut Curie, Service de chirurgie, 26 rue d'Ulm, 75248 Paris, France

${ }^{5}$ Hôpital de Hautepierre, Service de chirurgie, 1 avenue Molière, 67200 Strasbourg, France

${ }^{6}$ Centre Alexis Vautrin, service de chirurgie, 6 avenue de Bourgogne, 54519 Vandoeuvre les Nancy, France

${ }^{7}$ Centre Oscar Lambret, Service de chirurgie, 3 rue Fréderic Combemale, 59000 Lille, France

${ }^{8}$ Institut régional du Cancer de Montpellier (ICM), Service de radiothérapie, 208 avenue des apothicaires, 34298 Montpellier, France

${ }^{9}$ Institut régional du Cancer de Montpellier (ICM), Service d'oncologie médicale, 208 avenue des apothicaires, 34298 Montpellier, France

${ }^{10}$ Insitut Bergonié, Service de chirurgie, 229 cours de l'Argonne, 33076 Bordeaux Cedex, France

${ }^{11}$ Centre Léon-Bérard, Service de chirurgie, 28 rue Laennec, 69373 Lyon Cedex, France

${ }^{12}$ Institut universitaire du cancer de Toulouse, Service de chirurgie, 1 avenue Irène Jollio-Curie, 31059 Toulouse Cedex 9, France

${ }^{13}$ Centre hospitalo-universitaire de Rennes, Service de chirurgie, 2 rue Henri Le Guilloux, 35033 Rennes, France

Auteur correspondant: Dr Sébastien Carrère E-mail: Sebastien.Carrere@icm.unicancer.fr 


\section{Introduction:}

Les sarcomes des tissus mous (STM) sont des tumeurs malignes mésenchymateuses rares dont l'incidence est estimée entre 5,6 et 5,9/100000 par an (1). Ils présentent une grande diversité pathologique, se développent à tout âge, et peuvent atteindre n'importe quelle région anatomique. Compte tenu de ces spécificités, l'organisation de la prise en charge des sarcomes s'est structurée en France sous la forme de deux réseaux interdépendants, le premier anatomopathologique (Réseau de Référence en Pathologie des Sarcomes et des viscères : RRePS) et le second clinique (Réseau NetSarc). Ces réseaux s'expriment au sein du Groupe Sarcome Français - Groupe d'Etude des Tumeurs Osseuses (GSF-GETO) qui fédère l'ensemble des centres experts qui prennent en charge cette pathologie complexe. Dans une étude récente portant sur plus de 12000 patients, il est montré que cette organisation en réseaux en France a été un progrès majeur dans la prise en charge des sarcomes (2).

Les sarcomes du tractus urogénital compte pour moins de $5 \%$ de ces tumeurs et moins de $2 \%$ de l'ensemble des cancers urologiques. Le cordon spermatique est une localisation préférentielle puisqu'il représente un tiers des sarcomes urologiques chez l'homme (3). La prise en charge des sarcomes du cordon spermatique (SCS) est souvent inappropriée, en rapport avec une errance diagnostique initiale, une chirurgie inadaptée ainsi qu'à la méconnaissance de cette pathologie augmentant significativement le risque de récidive et grevant le pronostic des patients.

\section{Présentation clinique et démarche diagnostique}

Le sarcome du cordon spermatique (SCS) se présente communément par la présence d'une tuméfaction inguinoscrotale unilatérale augmentant progressivement de taille. L'examen clinique authentifie la présence d'une masse plus ou moins indurée, unilatérale, irréductible et indolore faisant suspecter le diagnostic. II peut également s'agir que d'une simple gêne voir d'une découverte fortuite lors d'une intervention chirurgicale pour cure de hernie. C'est dans ce dernier cas de figure que le risque de morcellation tumorale est le plus élevé (4).

Toute suspicion clinique de sarcome du cordon spermatique doit impérativement conduire à la réalisation d'une imagerie par résonnance magnétique (IRM). Elle est recommandée en première intention $(5,6)$ et permet de préciser la nature de la tuméfaction, sa taille, sa topographie, ses rapports anatomiques et d'éliminer les diagnostics différentiels. L'absence d'extension intra abdominale doit également être vérifiée (Figure 1).

La preuve histologique est apportée par la réalisation d'une biopsie percutanée coaxiale de large calibre (14 ou 16 gauges) sous contrôle radiologique. L'indication de biopsier est prise de façon collégiale en réunion de concertation pluridisciplinaire (RCP) régionale spécialisée dans la prise en charge des sarcomes. Le binôme radiologue-chirurgien précisera la voie d'abord biopsique qui sera emportée dans la future voie d'abord chirurgicale $(5,6)$. Cette 
biopsie est relue systématiquement par un pathologiste expert membre du réseau RRePS. L'instauration de cette double lecture gratuite établit en 2009 par l'Institut National du cancer (INCa) a diminué significativement le nombre d'erreurs diagnostiques et contribué ainsi à l'amélioration de la prise en charge de ces tumeurs (7).

Le liposarcome est le type histologique le plus représenté (46 à 62\%) chez l'adulte pour cette localisation. Il est suivi du léiomyosarcome (19 à $21 \%$ ) et enfin du rhabdomyosarcome (9 à $13 \%)(3,4,8)$. Cette répartition est différente chez l'enfant avec une nette prédominance du rhabdomyosarcome, de moins bon pronostic, justifiant souvent un traitement oncologique multimodal.

Les diagnostics différentiels de sarcome du cordon spermatique sont nombreux. II s'agit dans $80 \%$ des cas de tumeur bénigne, la plus fréquente étant le lipome du sujet âgé. II doit être distingué du liposarcome par la biopsie préopératoire avec recherche de l'amplification de MDM2 en biologie moléculaire. II peut également être question d'une autre affection maligne (cancer du testicule, lymphome ou localisation ganglionnaire secondaire) ou non tumorale (hernie inguinale, torsion ou hématome) (9). Par ailleurs, le cordon spermatique peut également être le site d'extension d'un sarcome primitif du rétropéritoine.

Un bilan d'extension à distance avec scanner thoracique est enfin réalisé, particulièrement chez l'adulte jeune ou en présence d'un léiomyosarcome.

\section{Aspects thérapeutiques et facteurs pronostiques}

La chirurgie est la pierre angulaire du traitement des sarcomes en général et particulièrement des SCS. Le principal critère de qualité est d'effectuer une exérèse monobloc de la tumeur avec des marges circonférentielles saines. Elle doit être réalisée par un chirurgien ayant l'expertise des problématiques liées à la chirurgie des sarcomes (gestion de la marge, prévention du risque de fragmentation tumorale, expérience en exérèse multi viscérale et en chirurgie de reconstruction). La planification de la chirurgie, incluant le " timing " de l'intervention et l'étendue de l'exérèse, diminuent significativement les risques de morcellation et d'exérèse incomplète qui ont le même pronostic péjoratif (10).

Une chimiothérapie néo adjuvante, bien qu'elle soit encore débattue (11), est discutée en présence d'un SCS nécessitant une chirurgie mutilante ou en présence d'une histologie laissant présager d'une chimiosensibilité (un rhabdomyosarcome par exemple). L'objectif escompté est une diminution du volume tumoral et une meilleure délimitation lésionnelle, favorisant ainsi l'exérèse (12).

La survie des SCS est favorable lorsqu'on compare à l'ensemble des STM. Radelli et al.(4) rapporte une survie spécifique à 5 ans de $92 \%$ pour une cohorte de 82 patients et un suivi médian de 36 mois. Le taux de récidive locale est de $26 \%$, avec comme facteur pronostic la 
qualité de l'exérèse chirurgicale $(p=0,025)$. Le taux de métastases à distance est de $24 \%$, impacté par le type histologique $(p<0,01)$, le grade tumoral élevé $(p<0,01)$ et par la réalisation d'une chimiothérapie adjuvante $(p=0,01)(4)$. Les mêmes facteurs pronostiques sont retrouvés dans la littérature qui comportent uniquement des séries rétrospectives $(3,4,8)$.

L'intérêt d'un traitement adjuvant est discuté en RCP spécialisée avec les résultats histologiques définitifs. L'hétérogénéité des cohortes rétrospectives n'autorise pas de recommandations factuelles à la réalisation d'une chimiothérapie adjuvante $(3,4,8,13)$. Elle sera proposée au cas par cas en présence de facteurs de mauvais pronostic. La même approche est réalisée pour la radiothérapie adjuvante, qui n'a théoriquement pas d'impact sur la survie sans récidive (4). De plus, la localisation du SCS est à risque de toxicité élevée (dysfonction érectile, stérilité, syndrome de Lapeyronie, rectite et de cystite radique). Néanmoins, certaines études suggèrent un bénéfice potentiel en terme de contrôle local d'autant plus que les techniques récentes de radiothérapie conformationnelle par modulation d'intensité (RCMI) ou les techniques rotationnelles permettent de protéger davantage les organes adjacents et donc de limiter significativement la toxicité (14).

\section{Spécificités chirurgicales}

L'objectif est de réséquer le sarcome qui est localisé le plus souvent dans le cordon, le testicule et ses enveloppes. Cette intervention sera élargie à la paroi et aux organes de voisinage en fonction de la taille tumorale, du type histologique, du bilan morphologique préopératoire, des antécédents chirurgicaux et de la présence de matériel prothétique.

L'installation du patient doit permettre un abord inguinoscrotal, une voie abdominale si nécessaire et également envisager une reconstruction par lambeau (Figure 2). La voie d'abord est directe, large et sans contre-incision afin de limiter les risques de fragmentation tumorale. Elle est réalisée dans l'axe du trajet des fibres musculaires et emporte le trajet de biopsie radiologique (Figure 3 ).

En amont, le cordon est sectionné au-dessus du pôle supérieur de la tumeur qui peut se situer en intraabdominal le long des vaisseaux spermatiques. En aval, le testis est systématiquement réséqué car on observe des extensions tumorales à ce niveau ou le long de l'épididyme. Les muscles crémastériens sont systématiquement réséqués et parfois les muscles obliques au niveau du canal inguinal, à fortiori s'il s'agit d'une reprise après effraction dans canal inguinal. Les zones à risque de marginalité sont en dedans les corps caverneux et en dehors les vaisseaux fémoraux (Figure 4). Dans les formes localement évoluées, on commence par disséquer les vaisseaux fémoraux en sous-adventicielle ce qui correspond à la marge externe (Figure 5). Ils seront remplacés en cas de nécessité. 
L'atteinte ganglionnaire est rare dans les sarcomes ne justifiant pas de curage systématique (5). Néanmoins, il sera discuté s'il existe une suspicion d'envahissement morphologique, notamment pour certain type histologique comme le rhabdomyosarcome. L'envahissement viscéral sous-jacent, peu fréquent également, peut impliquer la vessie, l'uretère et le colon, augmentant alors le risque de morbidité post opératoire. L'envahissement osseux est exceptionnel pour cette localisation.

La reconstruction pariétale fait le plus souvent l'objet d'une raphie musculaire du tendon conjoint, puis d'une fermeture de l'aponévrose du muscle oblique externe. Ceci est valable pour les petites tumeurs, de localisation préférentiellement scrotale et lorsque l'exérèse chirurgicale est optimale d'emblée. En cas de "Whoops " initiale, de matériel prothétique, de volumineuse tumeur ou de localisation tumorale funiculaire, une reconstruction plus élaborée est nécessaire. Le lambeau de muscle gracilis est le lambeau de choix (Figure 6). Sa mobilisation supérieure est réalisée par l'abord inguinoscrotal en prenant soin de ne pas léser son pédicule principal. Une contre incision distale est ensuite réalisée en face médiale de cuisse pour sectionner son tendon. Il est enfin fixé aux structures musculoaponévrotiques résiduelles puis renforcé par l'adjonction d'une plaque de tissu résorbable si nécessaire. Pour les défects les plus importants, notamment lorsque l'arcade crurale a été réséquée, la réalisation d'un lambeau de Taylor controlatéral ou d'un lambeau libre sera discutée.

En fin d'intervention et selon les recommandations du réseau Netsarc, la pièce opératoire est envoyée orientée au laboratoire d'anatomopathologie et un compte-rendu standardisé est créé, faisant apparaitre l'ensemble des critères de qualité de l'exérèse des sarcomes des tissus mous $(10,15)$. Le patient sera revu en postopératoire afin d'apprécier l'état de cicatrisation (Figure 7) et pour lui annoncer sa prise en charge ultérieure.

\section{Conclusion :}

La prise en charge des sarcomes du cordon spermatique en France doit faire l'objet d'une approche pluridisciplinaire dédiée et formalisée par les RCP spécialisées régionales du réseau Netsarc. L'orientation de ces patients, qui nécessitent le plus souvent des chirurgies complexes, doit se faire vers un chirurgien entrainé à opérer les sarcomes permettant ainsi d'améliorer significativement la qualité de l'exérèse et par conséquent leur survie. 


\section{Liste des figures :}

Figure 1: Rhabdomyosarcome du cordon spermatique gauche en IRM coupe transversale séquence T1 injecté au gadolinium

Figure 2 : Installation chirurgicale pour exérèse d'un sarcome du cordon spermatique

Figure 3 : Voie d'abord chirurgicale inguinoscrotale emportant le trajet de biopsie

Figure 4 : Orchidectomie gauche totale et éxérèse du cordon spermatique en monobloc

Figure 5 : Vue finale après exérèse

Figure 6: Reconstruction de l'arcade crurale par lambeau de muscle gracilis gauche après éxérèse élargie d'un sarcome du cordon spermatique

Figure 7 : Cicatrisation à J20 de l'exérèse élargie d'un sarcome du cordon spermatique 


\section{Références:}

1. Stiller CA, Trama A, Serraino D, Rossi S, Navarro C, Chirlaque MD, et al. Descriptive epidemiology of sarcomas in Europe: report from the RARECARE project. Eur J Cancer. 2013 Feb;49(3):684-95.

2. Blay J-Y, Soibinet P, Penel N, Bompas E, Duffaud F, Stoeckle E, et al. Improved survival using specialized multidisciplinary board in sarcoma patients. Ann Oncol. 2017 Nov 1;28(11):2852-9.

3. Coleman J, Brennan MF, Alektiar K, Russo P. Adult spermatic cord sarcomas: management and results. Ann Surg Oncol. 2003 Jul;10(6):669-75.

4. Radaelli S, Desai A, Hodson J, Colombo C, Roberts K, Gourevitch D, et al. Prognostic factors and outcome of spermatic cord sarcoma. Ann Surg Oncol. 2014 Oct;21(11):3557-63.

5. ESMO / European Sarcoma Network Working Group. Soft tissue and visceral sarcomas: ESMO Clinical Practice Guidelines for diagnosis, treatment and follow-up. Ann Oncol. 2012 Oct;23 Suppl 7:vii92-99.

6. Honoré $C$, Méeus $P$, Stoeckle $E$, Bonvalot $S$. Soft tissue sarcoma in France in 2015: Epidemiology, classification and organization of clinical care. J Visc Surg. 2015 Sep;152(4):223-30.

7. Neuville A, Coindre J-M. [Sarcomas, example of a pathologist network organization]. Bull Cancer (Paris). 2013 Dec;100(12):1275-81.

8. Rodríguez D, Barrisford GW, Sanchez A, Preston MA, Kreydin El, Olumi AF. Primary spermatic cord tumors: disease characteristics, prognostic factors, and treatment outcomes. Urol Oncol. 2014 Jan;32(1):52.e19-25.

9. Pecoux F, Ballereau C, Biserte J. Tuméfactions du cordon spermatique. EMC (Elsevier Masson SAS, Paris), Urologie. 2010;18-652-A-10.

10. Bonvalot $S$, Tzanis $D$, Ferron $G$, Toufik Bouhadiba $M$, Meeus $P$, Ducimetière $F$, et al. Les critères de qualité de la prise en charge chirurgicale des sarcomes des tissus mous. Oncologie. 2016;18:211-5.

11. Rothermundt C, Fischer GF, Bauer S, Blay J-Y, Grünwald V, Italiano A, et al. Pre- and Postoperative Chemotherapy in Localized Extremity Soft Tissue Sarcoma: A European Organization for Research and Treatment of Cancer Expert Survey. The Oncologist. 2017 Nov 30

12. Grabellus F, Podleska LE, Sheu S-Y, Bauer S, Pöttgen C, Kloeters C, et al. Neoadjuvant treatment improves capsular integrity and the width of the fibrous capsule of high-grade soft-tissue sarcomas. Eur J Surg Oncol. 2013 Jan;39(1):61-7.

13. Bachmann R, Rolinger J, Girotti P, Kopp HG, Heissner K, Amend B, et al. Liposarcoma of the Spermatic Cord: Impact of Final Surgical Intervention-An Institutional Experience. Int J Surg Oncol. 2016;2016:4785394. 
14. Cerda T, Martin É, Truc G, Créhange G, Maingon P. Safety and efficacy of intensity-modulated radiotherapy in the management of spermatic cord sarcoma. Cancer Radiother 2017 Feb;21(1):1620.

15. Stoeckle E, Michot A, Henriques B, Sargos P, Honoré C, Ferron G, et al. [Surgery for soft-tissue sarcomas of the limbs and trunk wall]. Cancer Radiother. 2016 Oct;20(6-7):657-65. 


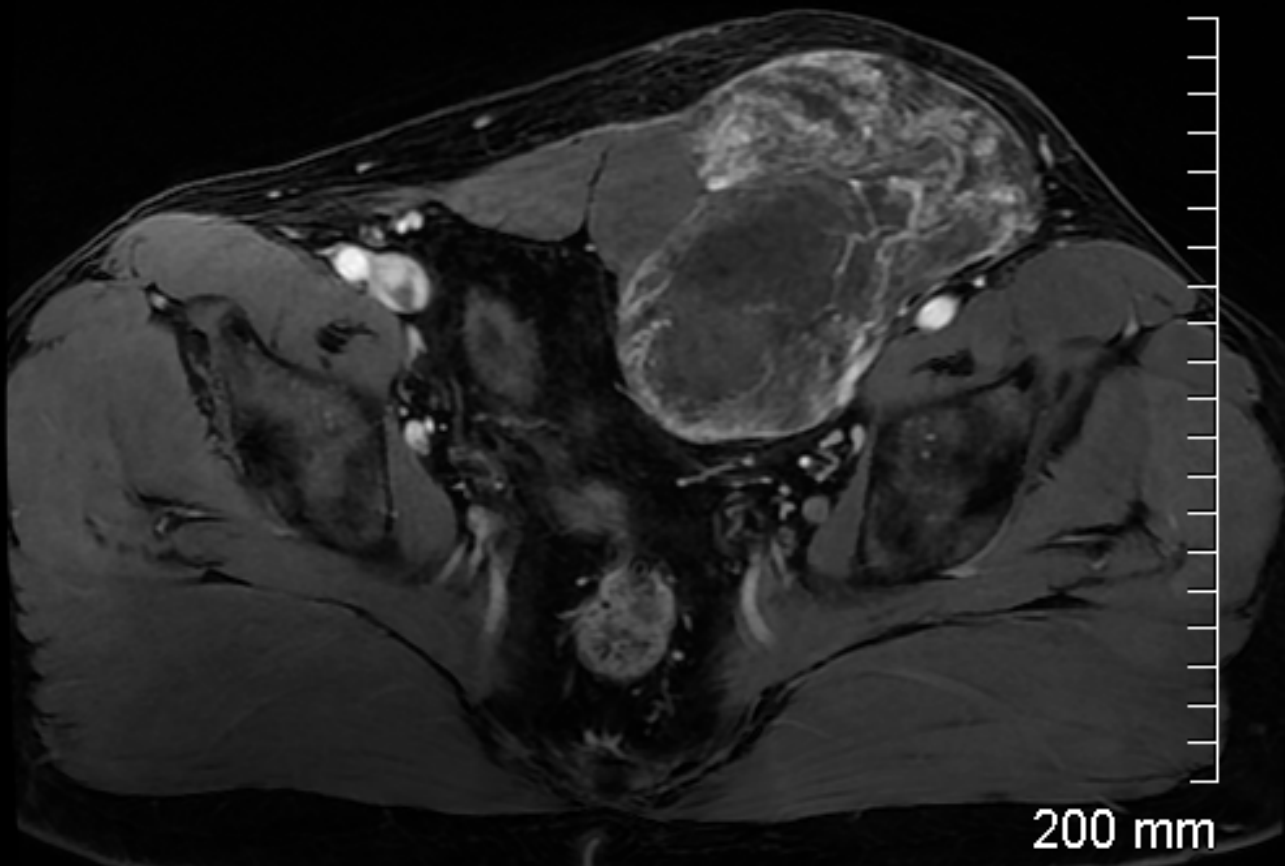




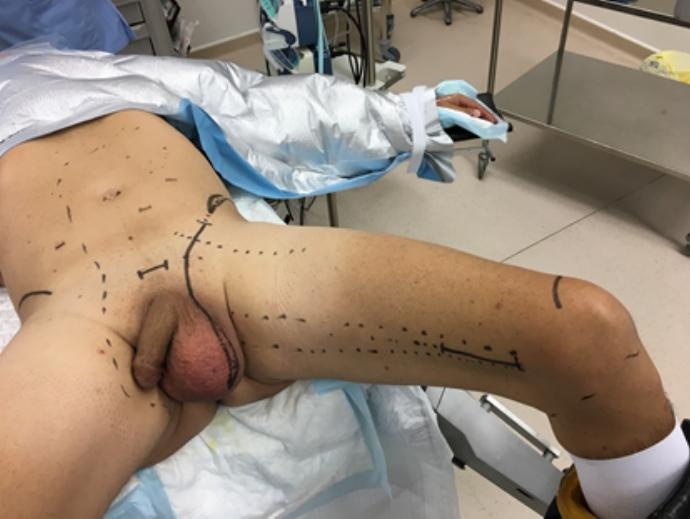




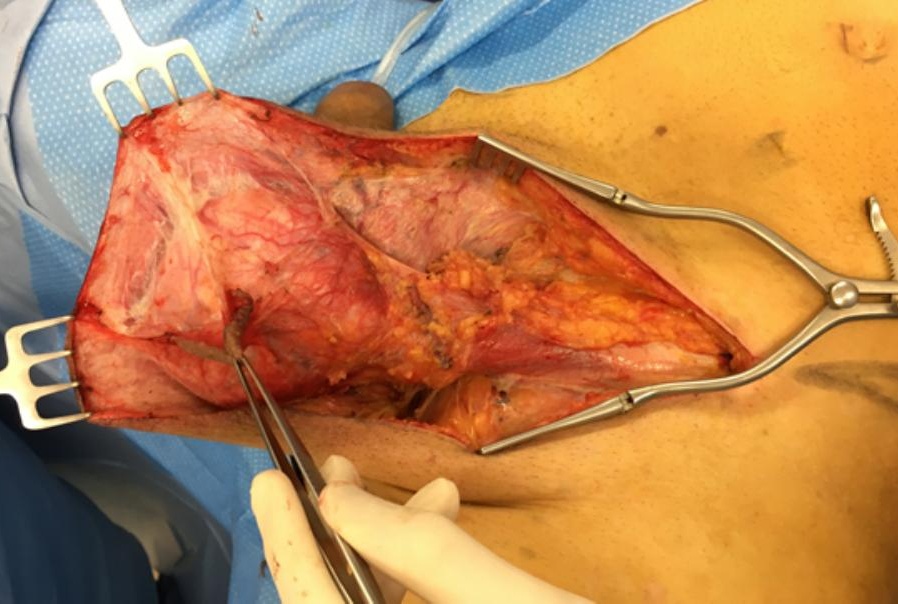




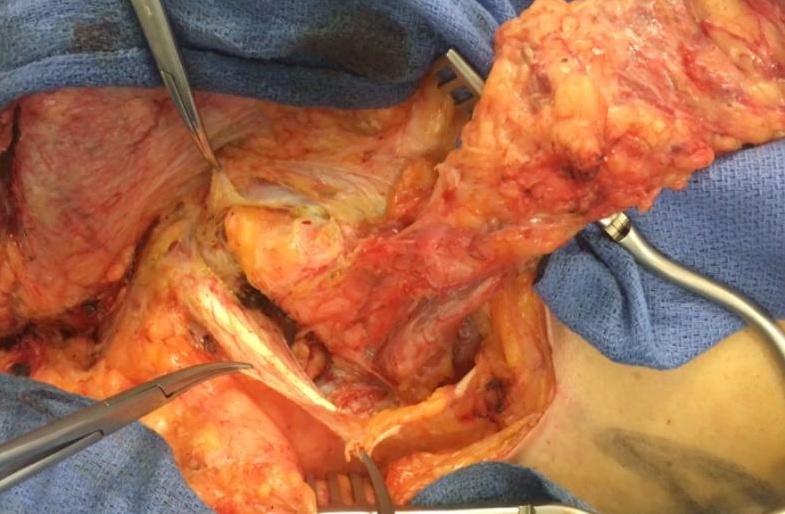




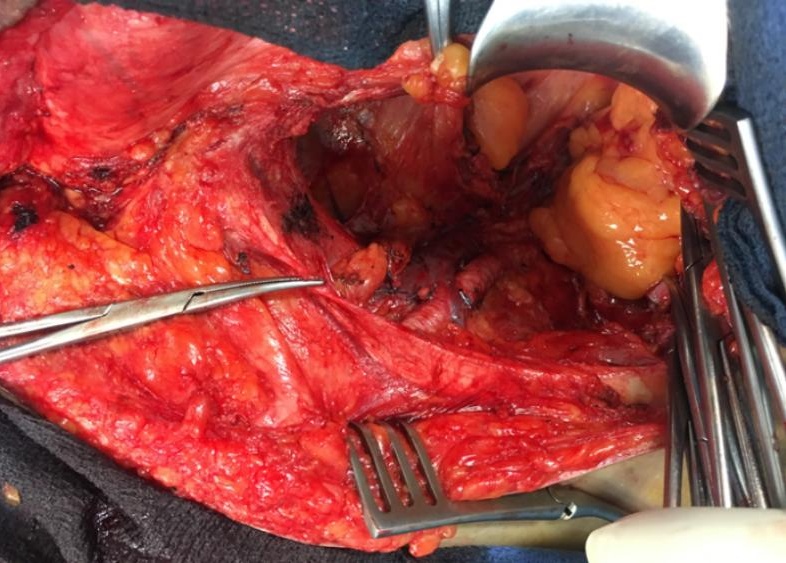




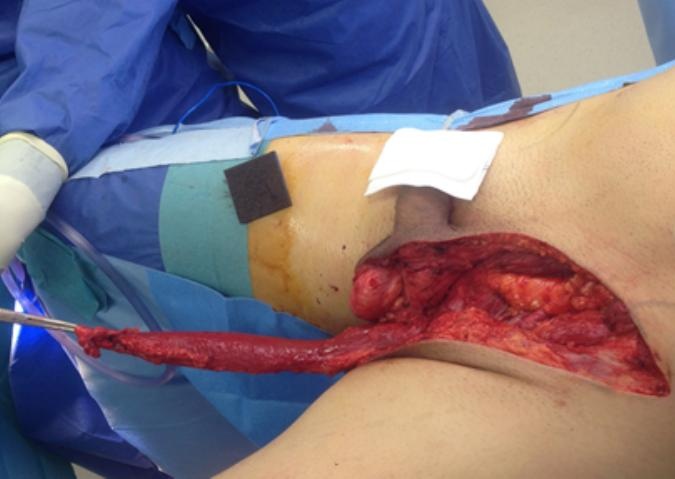




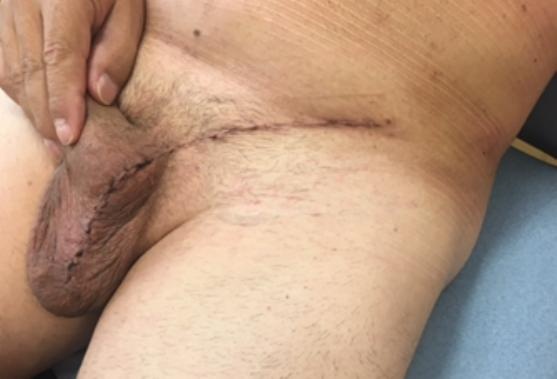

